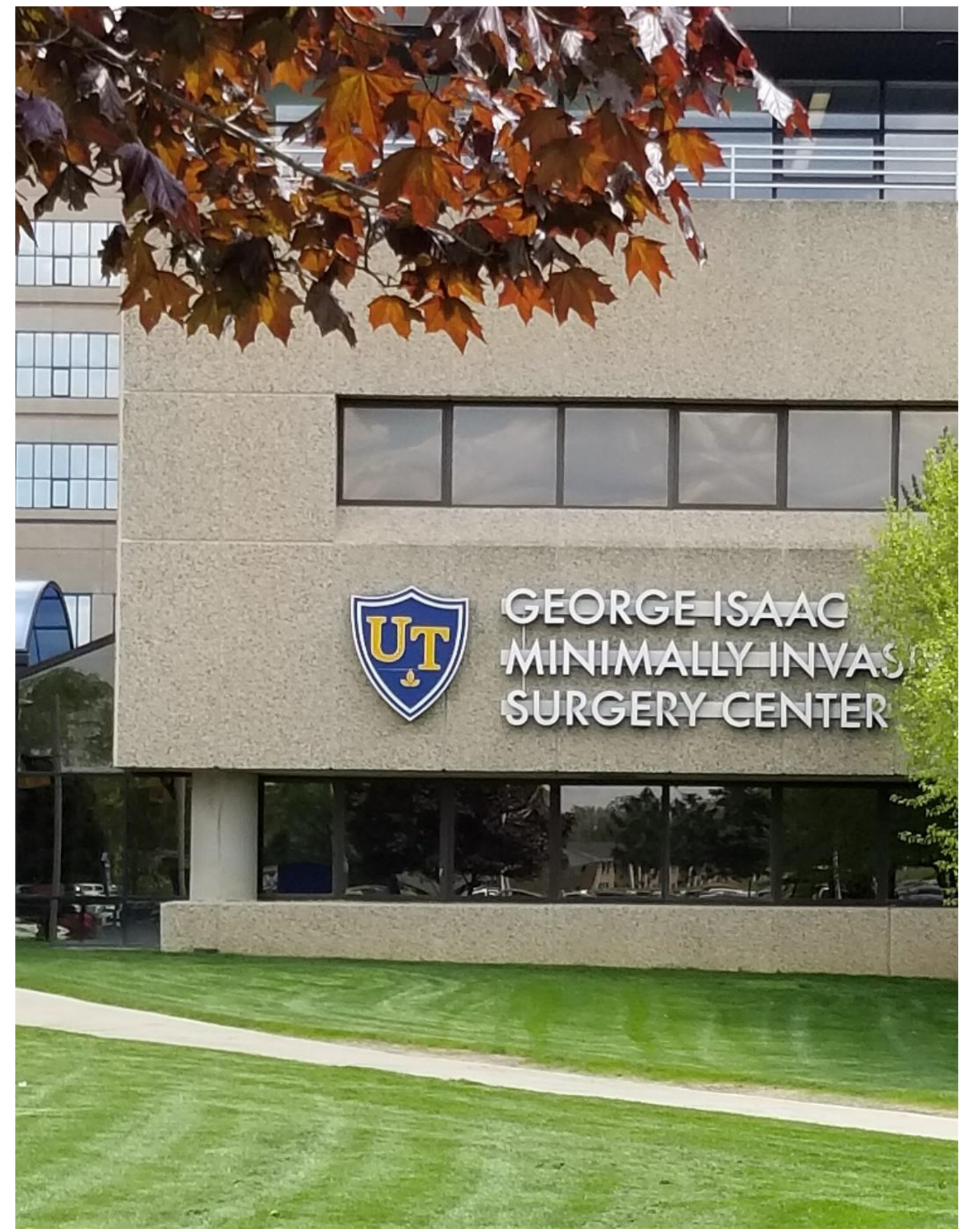




\title{
Performance of Molecular Breast Imaging as an Adjunct Diagnostic Tool
}

\author{
Robin B. Shermis ${ }^{a}, 1$ Roberta E. Redfern ${ }^{b}$, John Bazydlo ${ }^{b}$ Gabriel Naimy ${ }^{b}$ Haris Kudrolli ${ }^{c}$ John Chen ${ }^{d}$
}

${ }^{a}$ ProMedica Breast Care, ProMedica Toledo Hospital, Toledo, OH 43606, USA, ${ }^{b}$ ProMedica Research, ProMedica Toledo Hospital, Toledo OH, USA, ${ }^{c}$ Sun Nuclear Corporation, Melbourne, FL, and ${ }^{d}$ Department of Mathematics and Statistics, Bowling Green State University, Bowling Green OH, USA

\begin{abstract}
Purpose: The aim was to retrospectively assess the performance of molecular breast imaging (MBI) as an adjunct diagnostic tool when symptoms could not be explained by conventional imaging, or when mammography or ultrasound findings were equivocal. Methods: The analysis was comprised of women who underwent further testing with MBI after diagnostic mammography and/or targeted ultrasound. Outcome measures included sensitivity, specificity, positive, and negative predictive values. Receiver-operating characteristic (ROC) curve was constructed and analyzed as a performance measure. Results: In 301 women with a complete follow up data, $18(6.0 \%)$ were diagnosed with cancer. MBI detected cancer in 16 subjects; two interval cancers occurred. 15 of the 16 cancers detected by MBI were invasive. Overall sensitivity of $\mathrm{MBI}$ in this sample was $88.9 \%(95 \% \mathrm{Cl} 65.6$ 98.6), with $97.5 \%$ specificity ( $95 \% \mathrm{Cl} 95.0$ - 99.0). Positive predictive value (PPV) was $69.6 \%$, while negative predictive value for recall (NPV) was calculated as $99.3 \%$. ROC curves demonstrated excellent performance (area under the curve $=0.933$ ). Conclusions: $\mathrm{MBI}$ is a valuable diagnostic tool for further evaluation or to guide management when conventional imaging is incomplete. The majority of tumors in this study were invasive carcinomas with node negative status.
\end{abstract}

breast | invasive carcinomas | molecular imaging | diagnostic tool |

D igital mammography is the primary imaging modality for breast cancer screening and diagnostic workup of breast lesions; the technique has made significant contributions towards reducing mortality rates (1-3). However, mammography has limitations in dense breast tissue, postsurgical scar tissue, and contracted breast implants. Mammography may not be well-suited for the diagnosis of isodense and/or slow growing cancers $(4,5)$. Adjunct modalities such as targeted ultrasound are often used to correlate to mammography in cases where images are not conclusive or do not provide enough information about a potential lesion $(6,7)$.

Ultrasound differentiates tissue types based on morphology and echo pattern and can significantly improve characterization of abnormalities when used in conjunction with mammography (8). Sonography is frequently utilized as a problem-solving tool in breast imaging. Targeted ultrasound has been reported to improve detection of tumors in clinically indicated cases but can be subject to significant inter-operator variability $(9,10)$. Breast MRI can also be used for resolution of inconclusive imaging (11). Though breast MRI has performed well, (12-14) it is not suitable for all patients due to numerous possible contraindications such as implanted devices, claustrophobia or allergy to contrast, prohibitive cost, and restricted payer reimbursements (15). Due to the shortcomings of current supplemental modalities such as low specificity, the use of scintimammography, specifically molecular breast imaging (MBI), for screening and diagnostic purposes was revisited, and MBI has evolved.

Scintimammography involves the use of a radiotracer such as ${ }^{99 m}$ Tc-sestamibi, which is preferentially taken up by hypermetabolic breast cancer cells (16-18). Initially, the technique suffered from intrinsically low resolution and required a relatively high dose of ${ }^{99 m}$ Tc-sestamibi (19). These limitations have been overcome by MBI (20), which employs two separate semiconductor gamma cameras to construct high resolution images. The breast is placed in light compression (about $5 \mathrm{lbs}$ ) between two such detectors enabling high resolution, functional imaging of the entire breast with less than $300 \mathrm{MBq}$ administered dose (21). Our breast care center adopted this technique in 2011 and has performed over 10,000 MBI examinations since implementation. In the majority of these cases, MBI has been used in the supplementary screening of women with dense breasts (22). This study aims to evaluate the use of MBI as an adjunct diagnostic tool (problem solver) in patients where conventional imaging provided inconclusive results.

\section{Materials and Methods}

This is a retrospective review of patients who underwent MBI

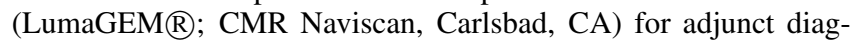
nostic imaging between April, 2011 and August, 2014 at ProMedica Breast Care Center. Women aged 25-90 years who presented with breast symptoms (focal pain, nipple discharge, and/or palpable lump) or were called back to further evaluate an asymmetry, calcifications, or masses on mammography on $2 \mathrm{D}$ digital diagnostic mammography (Hologic, Bedford, MA) without a sonographic correlate underwent diagnostic MBI and were eligible for inclusion in this review. Radiologist rating of mammography were BI-RADS 0-3 (indeterminate, benign, or probably benign). This study was approved by the ProMedica Institutional Board Review; written informed consent was waived.

Participants were injected with $300 \mathrm{mBq}(8 \mathrm{mCi})$ of $99 \mathrm{mTc}-$ sestamibi intravenously approximately 5 minutes prior to imaging. Bilateral mediolateral oblique (MLO) and craniocaudal (CC) views were collected for each participant under light compression. MBI images were interpreted by dedicated breast radiologists and assigned a BI-RADS score between 0 and 6; MBI BI-RADS categories parallel those used in mammography $(23,24)$. MBI BIRADS 0-3 were considered test negative, whereas BI-RADS 4 and

All authors contributed to this paper. ${ }^{1}$ To whom correspondence should be sent: rshermis@bex.net

Some authors declare conflict of interest. Submitted: 08/26/2019, published: 10/04/2019.

Freely available online through the UTJMS open access option 
5 were considered test positive. Women with MBI BI-RADS categorized as 6 (confirmed malignancy) were excluded from analysis. Women with positive MBI results underwent targeted ultrasoundguided biopsy; if the lesion was not visible under ultrasound, stereotactic- or MRI-guided biopsy was pursued. False positive cases were recommended to undergo follow-up mammography at 6 months, returning to annual screening mammography in the case of normal results. For those with dense or complex mammograms, biennial MBI is recommended. Test negative cases were recommended to undergo annual mammography.

\section{Statistical Analysis}

Only women whose diagnostic mammography examination was completed within 100 days of index MBI were included. The positive reference standard was defined as histopathologic diagnosis of breast cancer, whereas negative reference standard was defined as negative biopsy results following index MBI exam or negative follow-up mammographic examination occurring at least 330 days following index MBI exam. Participants without a complete reference standard were excluded from analysis. Cancers detected in any participant less than 365 days after negative index MBI examination were considered interval cancers. Descriptive statistics characterized the study population; sensitivity, specificity, positive and negative predictive values, cancer detection rate, and biopsy rate were calculated utilizing only patients with a complete reference standard. Breast density category and age were collected as these may impact risk of breast cancer and confound results. The area under the ROC curve (AUC) was calculated as an overall measure of the predictive power for MBI $(25,26)$. The effect of MBI in breast cancer detection is evaluated by the odds ratio estimation in the context of a logistic regression. Confidence intervals were calculated based on Wald statistics. All analyses were completed using SAS version 9.2 (SAS Institute Inc., Cary, NC, USA).

\section{Results}

In total, 367 women met inclusion criteria; of these, 66 of these had no additional follow up or imaging information available after MBI such that 301 included patients had a complete reference standard available for analysis. The mean age of included subjects was $49.8 \pm 11.2$ years (range $25-80$, Table 1 ). The ethnic and racial composition of the population is presented in Table 1 . The majority had heterogeneously or extremely dense breasts $(260 / 301,86.4 \%)$. Performance characteristics of MBI are presented in Table 2. Of 301 included patients, 18 (5.98\%) were ultimately diagnosed with cancer; 16 of these were detected with MBI yielding a sensitivity of $88.9 \%$ (95\% CI 65.3 - 98.6). In this sample, 7 false positive MBI studies were observed, resulting in $97.5 \%$ specificity (95\% CI 95.0 99.0). Positive and negative predictive values are presented in Table 2. Importantly, due to the small sample size of positive results, the lower limit for estimates of both sensitivity and PPV are low compared to the point estimates.

The data shows the strong predictive power of MBI as a diagnostic tool using a logistic regression model $(\mathrm{p}<0.0001)$. The overall predictive power is 0.933 , measured by the AUC of the estimated ROC curve, a plot between sensitivity and 1-specificity of the data. At $95 \%$ confidence level, MBI serves as a strong predictor of cancer diagnosis with an estimated odds ratio of $8.01(95 \%$ CI $3.780-17.360$ ), suggesting the odds of breast cancer increases by about 8 - fold among those women with an increasing MBI BIRADS category (Figure 1, Table 3 ). Of the 16 patients with cancer detected by MBI, 15 (93.8\%) were invasive tumors; histopathology demonstrated ductal carcinoma in situ in 1 patient $(6.25 \%)$.

Table 1. Study participant characteristics

\begin{tabular}{cc}
\hline \hline & \\
Participant characteristics & $\mathrm{n}=301$ \\
\hline Age at Index MBI, years $\pm S D$ (range) & $49.8 \pm 11.2(25-80)$ \\
Race, $\mathbf{n}$ ( \%) & \\
Asian & $1(0.3 \%)$ \\
Hispanic & $12(4.0 \%)$ \\
White & $6(2.0 \%)$ \\
Other & $266(88.4 \%)$ \\
Unknown & $6(2.0 \%)$ \\
& $10(3.3 \%)$ \\
Breast Density, n ( \% ) & \\
Almost entirely fatty & $1(0.3 \%)$ \\
Scattered fibroglandular densities & $40(13.3 \%)$ \\
Heterogeneously Dense & $160(53.2 \%)$ \\
Extremely Dense & $100(33.2 \%)$ \\
& \\
Mammogram BI-RADS n (\%) & $163(54.2 \%)$ \\
BI-RADS 0 & $47(15.6 \%)$ \\
BI-RADS 1 & $27(9.0 \%)$ \\
BI-RADS 2 & $64(21.2 \%)$ \\
BI-RADS 3 & \\
&
\end{tabular}

Table 2. Performance Characteristics of Molecular Breast Imaging at participant level

\begin{tabular}{ccc}
\hline \hline Parameter & $\begin{array}{c}\text { Number of patients } \\
\text { vs. total }\end{array}$ & $\begin{array}{c}\text { Estimate } \\
(95 \% \text { CI) }\end{array}$ \\
\hline Cancer prevalence rate & $18 / 301$ & $5.98(3.58-9.29)$ \\
Sensitivity (\%) & $16 / 18$ & $88.9(65.3-98.6)$ \\
Specificity (\%) & $276 / 283$ & $97.5(95.0-99.0)$ \\
Biopsy rate (\%) & $23 / 301$ & $7.64(4.91-11.3)$ \\
PPV (\%) & $16 / 23$ & $69.6(51.9-82.9))$ \\
\hline
\end{tabular}

Table 3. Logistic regression of factors relating to breast cancer diagnosis and receiver operator characteristic analysis with AUC $(\mathrm{c}=0.933)$

Odds Ratio Estimates

\begin{tabular}{cccc}
\hline Effect & $\begin{array}{c}\text { Point } \\
\text { estimate }\end{array}$ & p-value & $\begin{array}{c}95 \% \text { Wald } \\
\text { confidence limits }\end{array}$ \\
\hline MBI result & 8.101 & $<0.0001$ & $3.780-17.360$ \\
Density & 2.190 & 0.1725 & $0.710-6.756$ \\
Age & 1.059 & 0.0914 & $0.991-1.131$ \\
\hline
\end{tabular}


Table 4. Tumor characteristics of cancers; true positives were detected on diagnostic MBI; false negatives were interval cancers occurring at 332 and 218 days after MBI examination

\begin{tabular}{ccccccccccc}
\hline \hline Pathology & $\begin{array}{c}\text { ER } \\
\text { status }^{f}\end{array}$ & $\begin{array}{c}\text { PR } \\
\text { status }^{g}\end{array}$ & $\begin{array}{c}\text { HER2/Neu } \\
\text { status }^{h}\end{array}$ & $\begin{array}{c}\text { Size } \\
(\mathrm{cm})\end{array}$ & Nodes & $\begin{array}{c}\text { Age } \\
\text { yrs. }\end{array}$ & $\begin{array}{c}\text { Breast } \\
\text { comp. } .^{i}\end{array}$ & $\begin{array}{c}\text { Breast } \\
\text { Risk } \\
(\%)\end{array}$ & $\begin{array}{c}\text { Mamm. } \\
\text { results }\end{array}$ & $\begin{array}{c}\mathrm{MBI}^{k} \\
\text { results }\end{array}$ \\
\hline
\end{tabular}

\section{True Positives}

\begin{tabular}{|c|c|c|c|c|c|c|c|c|c|c|}
\hline $\mathrm{IDC}^{a}$ & Positive & Positive & Equivocal & 0.9 & Negative & 56 & $\mathrm{C}$ & Left & 10.00 & 0 \\
\hline $\operatorname{DCIS}^{b}$ & Negative & Negative & N/A & N/A & Negative & 31 & $\mathrm{D}$ & Left & 15.10 & 0 \\
\hline IDC & Positive & Positive & Negative & 0.6 & Unknown & 78 & B & Left & 8.20 & 0 \\
\hline IDC & Positive & Positive & Negative & 0.8 & Negative & 80 & B & Right & 2.00 & 0 \\
\hline $\mathrm{ILC}^{c} / \mathrm{LCIS}^{d}$ & Positive & Positive & Negative & 2.1 & Negative & 47 & $\mathrm{C}$ & Right & 7.90 & 3 \\
\hline IDC/DCIS & Positive & Positive & Negative & 1.7 & Positive & 53 & $\mathrm{C}$ & Left & 9.60 & 3 \\
\hline IDC & Positive & Positive & Negative & 0.8 & Negative & 51 & D & Right & 15.55 & 0 \\
\hline IDC/DCIS & Positive & Negative & Equivocal & $1.1,1.3$ & Positive & 59 & $\mathrm{C}$ & Bilateral & 5.70 & 0 \\
\hline IDC & Positive & Positive & Negative & 1.1 & Negative & 67 & B & Left & 6.48 & 0 \\
\hline IDC/DCIS & Positive & Positive & Negative & 2.2 & Negative & 42 & $\mathrm{D}$ & Left & 13.50 & 0 \\
\hline $\mathrm{IPC}^{e} / \mathrm{IDC}$ & Positive & Positive & N/A & $2.8,0.8$ & Negative & 73 & $\mathrm{C}$ & Bilateral & 4.80 & 0 \\
\hline IDC & Positive & Negative & Negative & 1.1 & Negative & 38 & $\mathrm{D}$ & Right & 11.60 & 0 \\
\hline IDC/DCIS & Positive & Positive & Negative & 0.5 & Negative & 68 & $\mathrm{C}$ & Right & 34.63 & 0 \\
\hline ILC & Positive & Positive & Equivocal & 3.3 & Positive & 47 & $\mathrm{C}$ & Right & 9.40 & 0 \\
\hline IDC & Positive & Positive & Negative & 1.2 & Negative & 73 & $\mathrm{C}$ & Right & 11.00 & 0 \\
\hline ILC & Positive & Positive & Negative & 2.0 & Positive & 65 & $\mathrm{C}$ & Left & 9.50 & 0 \\
\hline
\end{tabular}

False Negatives

$\begin{array}{cccccccccccc}\text { IDC } & \text { Positive } & \text { Positive } & \text { Positive } & 2.2 & \text { Negative } & 47 & \text { D } & \text { Left } & 7.31 & 0 & 1 \\ \text { IDC/DCIS } & \text { Negative } & \text { Negative } & \text { Negative } & 2.7,1.3 & \text { Negative } & 45 & \text { C } & \text { Left } & \text { N/A } & 3 & 1\end{array}$

a IDC - invasive ductal carcinoma, b DCIS - ductal carcinoma in situ, c ILC - invasive lobular carcinoma, d LCIS - lobular carcinoma in situ, e IPC - intracystic papillary carcinoma, f ER status - estrogen receptor status, g PR status - progesterone receptor status, h HER2/neu - human

epidermal growth factor receptor status. i breast composition: A - almost entirely fatty, B - scattered areas of fibroglandular density; C -

heterogeneously dense; D - extremely dense. j mammography results: reported as BI-RADS, k MBI results, reported as BI-RADS.

Two women had bilateral disease (12.5\%). Two interval cancers were not detected on MBI. The majority of tumors occurred in heterogeneously dense breasts $(86.4 \%$, Table 2$)$. In patients whose tumors were detected by MBI, 8 (50.0\%) tumors were less than 10 $\mathrm{mm}$. Furthermore, the majority of cancers detected by MBI presented with no involvement of the lymph nodes (Table 4).

\section{Discussion}

In this study, we found that MBI detected cancer in 16 of $18(88.9 \%)$ patients when conventional imaging was exhausted. 93.75\% (15/16) of patients with cancer detected by MBI were found to have invasive disease; the average tumor size detected by MBI was $1.35 \mathrm{~cm}$ (range $0.5-3.3 \mathrm{~cm}$ ). Importantly, the majority of cancers detected by MBI presented with node negative status (68.8\%).

Early studies of the use of MBI suggest sensitivity of approximately $85 \%-91 \%$ (20). A study published in 2011 reported a cumulative sensitivity of mammography with $\mathrm{MBI}$ in a screening popula- tion as approximately $91 \%$, with MBI's specificity being 93\%(27). Later studies showed that the three fold reduction in radiation dose did not negatively impact the sensitivity nor specificity $(21,28)$. The diagnostic performance characteristics calculated from this sample agrees well with previous reports, with a high sensitivity such that unnecessary biopsy can be avoided. Ultrasound and MRI are often used in the resolution of indeterminate mammograms and have demonstrated high sensitivity in dense breasts. The addition of each modality results in reductions in specificity as reported in the ACRIN 6666 trials (29). Meissnitzer et al reported that ultrasonography exhibited sensitivity of $99 \%$, however, the specificity was unsatisfactorily low at $20 \%$, similar to previous studies $(30,31)$. Moreover, in a diagnostic setting, ultrasonography was unable to resolve inconclusive mammography in nearly $40 \%$ of cases (32). In our study, only 4 (1.3\%) cases resulted in an MBI BIRADS 0 diagnosis requiring additional work-up with MRI.

Reports comparing BSGI and MRI have shown the techniques performed similarly in terms of sensitivity, however, results suggest less variable specificity of BSGI $(33,34)$. Furthermore, in a report from a community breast care center, MRI suggested similar sensi- 
tivity with lower specificity compared to BSGI (54\% vs. 73\%) (35). Based on this information, a study of direct comparison of diagnostic performance of MBI and MRI merits consideration, particularly because MBI is much less expensive, requires fewer resources to complete, has fewer contraindications, and is much quicker to interpret than MRI examinations.

Of particular importance is the extremely high negative predictive value (99.3\%) of MBI in this diagnostic context. These patients were experiencing symptoms such as pain or discharge, or had imaging findings that were not explained by mammography or ultrasound. In such situations, biopsy would be performed or the patient would be recommended to have follow up imaging in 6 months, which results in increased patient anxiety and unnecessary cost. Because the NPV is so high, radiologists and patients can be confident that a negative MBI result ensures that cancer is not present and further action is not needed, preventing needless worry and expense, as well as the potential risks associated with biopsy.

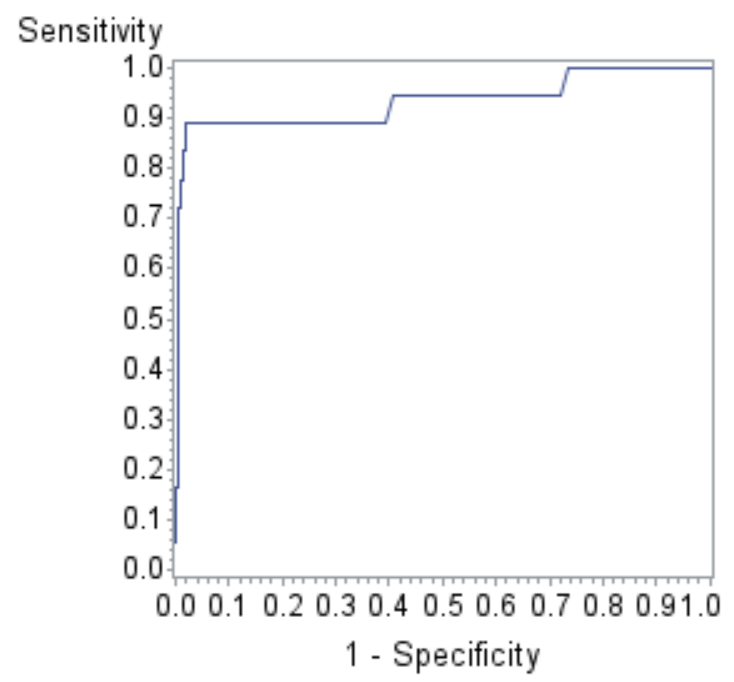

Figure 1: Logistic regression on MBI with receiver operator characteristic analysis, area under the curve $(A U C)(c=0.933)$. This is a statistical analysis exercise to gain insight into the robustness of $M B I$ findings. Here we change the threshold for positive findings from BIRADS 0 through 5 and observe the shape of the curve. AUC $>0.9$ signifies a robust/excellent test

This study is subject to a number of limitations. This is a singleinstitution study, albeit community based, and the wider application of this technology may help to validate or modify our reported re-

1. Bjurstam N, et al. (1997) The Gothenburg Breast Cancer Screening Trial: preliminary results on breast cancer mortality for women aged 39-49. Journal of the National Cancer Institute. Monographs (22):53-55.

2. Bjurstam N, et al. (1997) The Gothenburg breast screening trial: first results on mortality, incidence, and mode of detection for women ages 39-49 years at randomization. Cancer 80(11):2091-2099.

3. Jonsson H, Nystrom L, Tornberg S, \& Lenner P (2001) Service screening with mammography of women aged 50-69 years in Sweden: effects on mortality from breast cancer. Journal of medical screening 8(3):152-160.

4. Krecke KN \& Gisvold JJ (1993) Invasive lobular carcinoma of the breast: mammographic findings and extent of disease at diagnosis in 184 patients. AJR American journal of roentgenology 161(5):957-960.

5. Jones EA, Phan TD, Blanchard DA, \& Miley A (2009) Breast-specific gammaimaging: molecular imaging of the breast using $99 \mathrm{mTc}$-sestamibi and a small- sults. Due to its retrospective nature, it was not possible to locate all necessary data, particularly in some women who may have been diagnosed in our health system but underwent surgery at another facility. Moreover, because the study site is a referral center for multiple screening sites within a large integrated healthcare system, $18 \%$ of identified women did not have one year follow up data in our center. Additionally, while all women with inconclusive diagnostic imaging are recommended to undergo MBI in our center to further characterize suspicious lesions and/or direct management, it is not possible for us to determine the proportion who ultimately did undergo the test. Moreover, due to the small number of positive results in the cohort, the study may be underpowered to estimate sensitivity and positive predictive value as observed by the wide confidence intervals. Finally, we did not collect detailed information about ultrasound or other imaging performed prior to MBI, such that a direct comparison of the results of each imaging modality cannot be made.

\section{Conclusion}

Our study showed that MBI performed extremely well as an adjunct diagnostic tool in women where mammography and adjunct imaging were indeterminate. Our results support previous studies which estimate high sensitivity and specificity of MBI in the detection of breast cancer, even in women with dense breast tissue. Factors related to breast cancer are relatively complicate and intertwining. Such factors include breast density, hormone level, age, menopause, and use of estrogen. This technique detected small, invasive tumors requiring treatment, in most cases prior to the involvement of lymph nodes. This leads us to conclude that MBI is a valuable tool to gain diagnostic information when mammography results are lacking.

\section{Conflict of interest}

Shermis RB. is member of Scientific Advisory Board, Gamma Medica Inc., Carlsbad, CA 92010, USA. Redfern RE, Kudrolli H, Bazydlo J, Naimy G, Chen J, declare no conflict of interest.

\section{Authors' contributions}

RS - study conception and design, data collection and data interpretation; RR - study design, data collection, data analysis and interpretation; JB - data collection; GN - data collection; HK - study conception and design, data interpretation; JC - data analysis and interpretation. All authors participated in writing the manuscript and/or revising for important content and approved of final version.

\section{Acknowledgments}

The authors would like to thank Kristy Williams, RHIT, CTR and The ProMedica Cancer Registry for assistance in locating data. We would also like to thank Mr. Stephen Wanjiku, MS for assistance in data collection. 
10. Mendelson EB \& Tobin CE (1995) Critical pathways in using breast US. Radiographics : a review publication of the Radiological Society of North America, Inc 15(4):935-945.

11. Wernli KJ, et al. (2014) Patterns of breast magnetic resonance imaging use in community practice. JAMA internal medicine 174(1):125-132.

12. Kriege M, et al. (2004) Efficacy of MRI and mammography for breast-cancer screening in women with a familial or genetic predisposition. The New England journal of medicine 351(5):427-437.

13. Warner E, et al. (2004) Surveillance of BRCA1 and BRCA2 mutation carriers with magnetic resonance imaging, ultrasound, mammography, and clinical breast examination. Jama 292(11):1317-1325.

14. Leach MO, et al. (2005) Screening with magnetic resonance imaging and mammography of a UK population at high familial risk of breast cancer: a prospective multicentre cohort study (MARIBS). Lancet 365(9473):1769-1778.

15. Saslow D, et al. (2007) American Cancer Society guidelines for breast screening with MRI as an adjunct to mammography. CA: a cancer journal for clinicians 57(2):75-89.

16. Maublant $\mathrm{J}$, et al. (1996) Technetium-99m-sestamibi uptake in breast tumor and associated lymph nodes. Journal of nuclear medicine : official publication, Society of Nuclear Medicine 37(6):922-925.

17. Maublant JC, et al. (1993) In vitro uptake of technetium-99m-teboroxime in carcinoma cell lines and normal cells: comparison with technetium-99m-sestamibi and thallium-201. Journal of nuclear medicine : official publication, Society of Nuclear Medicine 34(11):1949-1952.

18. Cordobes MD, et al. (1996) Technetium-99m-sestamibi uptake by human benign and malignant breast tumor cells: correlation with mdr gene expression. Journal of nuclear medicine : official publication, Society of Nuclear Medicine 37(2):286289.

19. Brem RF, et al. (2008) Breast-specific gamma imaging as an adjunct imaging modality for the diagnosis of breast cancer. Radiology 247(3):651-657.

20. Hruska CB, et al. (2008) Scientific Impact Recognition Award: Molecular breast imaging: a review of the Mayo Clinic experience. American journal of surgery 196(4):470-476.

21. Rhodes DJ, et al. (2015) Journal club: molecular breast imaging at reduced radiation dose for supplemental screening in mammographically dense breasts. AJR. American journal of roentgenology 204(2):241-251.

22. Shermis RB, et al. (2016) Supplemental Breast Cancer Screening With Molecular Breast Imaging for Women With Dense Breast Tissue. AJR. American journal of roentgenology:1-8.
23. Conners AL, et al. (2012) Lexicon for standardized interpretation of gamma camera molecular breast imaging: observer agreement and diagnostic accuracy. European journal of nuclear medicine and molecular imaging 39(6):971-982.

24. Conners AL, et al. (2012) Gamma camera breast imaging lexicon. AJR. American journal of roentgenology 199(6):W767-774.

25. van Erkel AR \& Pattynama PM (1998) Receiver operating characteristic (ROC) analysis: basic principles and applications in radiology. European journal of radiology 27(2):88-94.

26. Obuchowski NA (2003) Receiver operating characteristic curves and their use in radiology. Radiology 229(1):3-8.

27. Rhodes DJ, Hruska CB, Phillips SW, Whaley DH, \& O'Connor MK (2011) Dedicated dual-head gamma imaging for breast cancer screening in women with mammographically dense breasts. Radiology 258(1):106-118.

28. Hruska CB, et al. (2015) Diagnostic workup and costs of a single supplemental molecular breast imaging screen of mammographically dense breasts. AJR. American journal of roentgenology 204(6):1345-1353.

29. Berg WA, et al. (2012) Detection of breast cancer with addition of annual screening ultrasound or a single screening MRI to mammography in women with elevated breast cancer risk. Jama 307(13):1394-1404.

30. Meissnitzer T, et al. (2015) Added value of semi-quantitative breast-specific gamma imaging in the work-up of suspicious breast lesions compared to mammography, ultrasound and 3-T MRI. The British journal of radiology 88(1051):20150147.

31. Skaane P \& Engedal K (1998) Analysis of sonographic features in the differentiation of fibroadenoma and invasive ductal carcinoma. AJR. American journal of roentgenology 170(1):109-114.

32. Zanello PA, et al. (2011) Breast ultrasound diagnostic performance and outcomes for mass lesions using Breast Imaging Reporting and Data System category 0 mammogram. Clinics 66(3):443-448.

33. Kim BS (2012) Usefulness of breast-specific gamma imaging as an adjunct modality in breast cancer patients with dense breast: a comparative study with MRI. Annals of nuclear medicine 26(2):131-137.

34. Edwards C, et al. (2013) Breast-specific gamma imaging influences surgical management in patients with breast cancer. The breast journal 19(5):512-519.

35. Johnson N, et al. (2014) Breast-specific gamma imaging is a cost effective and efficacious imaging modality when compared with MRI. American journal of surgery 207(5):698-701; discussion 701. 